${ }^{1}$ Servicio de Oftalmología. Hospital del Salvador. Santiago, Chile. anterno Medicina. Universidad de Los Andes. Santiago, Chile. bPrograma de formación de especialista en Oftalmología. Escuela de Postgrado, Facultad de Medicina, sede Norte, Universidad de Chile. Santiago, Chile.

Trabajo no recibió financiamiento. Los autores declaran no tener conflictos de interés.

Recibido el 17 de enero de 2018, aceptado el 19 de julio de 2018 .

Correspondencia a: Manuel Gallegos magallegos@miuandes.cl

\section{Experiencia de atención en red entre Hospital del Salvador y tres unidades de Atención Primaria Oftalmológica: Un modelo de salud pública para mejorar la atención oftalmológica}

\author{
ALEX JONES, MANUEL GALLEGOS ${ }^{a}$, \\ IGNACIO DÍAZ $^{\text {b }}$ RODRIGO DONOSO ${ }^{1}$
}

\section{A model of ophthalmological network care including primary care centers and a tertiary care hospital}

Background: Primary care units of ophtalmology (UAPO) were incorporated in 2003 into the healthcare system of the Ministry of Health, in response to the high demand for hospital care. Three of these primary care units were incorporated to provide a network care with the ophthalmology service of a tertiary care hospital. Aim: To report the public health impact of networking. Material and Methods: A descriptive-prospective study was carried out analyzing all the healthcare attentions carried out between June and August 2016. An epidemiological characterization of the sample was made. We recorded: clinical diagnoses, decisions, resolution of the clinical problem at the primary care or referral to the hospital. Results: A total of 2,096 ophthalmologic attentions were carried out in the three UAPOs during the study period. The main diseases attended were disorders of refraction, cataracts, glaucoma and diabetic retinopathy. The resolution index was $84 \%$ of attentions. Only $16 \%$ of attentions required referral and consisted mainly of cataracts (covered by a special health care system), diabetic retinopathy, capsulotomies, iridotomies, uveitis, pterygium and lacrimal duct obstruction. Conclusions: This model of care allowed an efficient management of the high demand for hospital referral. This is expressed in the high-resolution index and low derivation, resulting in a decrease in waiting lists at the tertiary level. This system should be implemented by other tertiary centers of our country.

(Rev Med Chile 2018; 146: 890-893)

Key words: Ophthalmology; Primary Health Care; Tertiary Healthcare.
A pesar de que el acceso a la salud visual en Chile ha mejorado en los últimos años, aún persisten largas listas de espera en los diferentes hospitales, tanto de la Región Metropolitana como de regiones a lo largo de nuestro país.

A raíz de este problema, y con el objetivo de acercar la atención de salud visual a la población, nace en 1960 la primera Unidad de Atención
Primaria en Oftalmología (UAPO), bajo el alero del Dr. Juan Arentsen Sauer y el equipo del Departamento de Oftalmología del Hospital San Juan de Dios (HSJD) ${ }^{1}$. Posteriormente, a partir del año 2003, estas unidades se incorporaron a la atención del Ministerio de Salud (MINSAL) en el marco del Programa de Resolutividad de la División de Atención Primaria (DIVAP). Se 
planteó, como uno de sus objetivos principales, la resolución de la patología refractiva, así como priorizar la derivación a nivel terciario con pertinencia y oportunidad. Desde aquel año, las UAPO han ido aumentando progresivamente en número de unidades y de atenciones y ya para el año 2016, el país contaba con 115 unidades de norte a sur, extendiendo, además, su cartera de prestaciones también a algunas patologías GES.

A partir del año 2014, dos UAPOs, la de Peñalolén y La Reina, sumadas a la UAPO de Ñuñoa, inaugurada en junio de 2016, han ofrecido sus servicios oftalmológicos mediante el establecimiento de una atención red en conjunto con el Servicio de Oftalmología del Hospital del Salvador (HdS).

\section{Propósito}

El propósito de este trabajo es dar a conocer la atención realizada por las UAPOs y el impacto en salud pública que ha significado el trabajo en red entre el Hospital del Salvador y las UAPOs de Peñalolén, La Reina y Ñuñoa.

\section{Material y Método}

Se efectuó un estudio descriptivo-prospectivo de todas las atenciones efectuadas entre junio y octubre de 2016 de las tres UAPOs mencionadas. Para ello, se hizo una caracterización epidemiológica de la muestra, analizando los datos en EpiData Software ${ }^{\circledR}$. Se registraron: número de identificación del paciente asignado según orden de registro, RUT, edad, fecha de atención, antecedentes mórbidos, agudeza visual con y sin corrección, tensión ocular. Se registró el examen físico ocular completo y, para cada atención, se registraron los diagnósticos y decisiones clínicas, según sea resolución a nivel de UAPO (alta o control periódico) o derivación al Hospital del Salvador. Todos los pacientes firmaron un consentimiento informado para publicación de sus datos clínicos y el estudio fue aprobado por los comités de ética del consultorio y del Hospital del Salvador.

En la UAPO, según evaluación del médico oftalmólogo o tecnólogo médico, pudiera generarse un hallazgo de problema de salud garantía explícita de salud (GES). Si así fuera, ambos profesionales deben realizar una solicitud de interconsulta (SIC), la cual debe contener datos relevantes básicos del paciente, y derivar al centro de referencia de nivel secundario correspondiente.
Catarata es aquella opacidad del cristalino que provoca una disminución de la agudeza visual gradual e indolora que no corrige con agujero estenopéico, siendo GES cuando se presenta una visión menor a 0,3. La confirmación diagnóstica la realiza el médico oftalmólogo, descartando previamente otra causa de agudeza visual disminuida ${ }^{2}$. La sospecha de glaucoma se presenta ante un incremento de la excavación de la papila del nervio óptico, pérdida del anillo neuroretinal, atrofia peripapilar asociados o no a una presión intraocular elevada. La confirmación diagnóstica la realiza el especialista mediante una serie de pruebas complementarias, como son la campimetría, paquimetría, tomografía de coherencia óptica (OCT) de papila y gonioscopía ${ }^{3}$. Finalmente, la presencia de retinopatía diabética se basa fundamentalmente en ciertos hallazgos al fondo de ojo, según los cuales podemos dividirla en niveles como pacientes sin retinopatía, retinopatía no proliferativa (leve, moderada y severa) o retiniopatía proliferativa (con o sin signos de alto riesgo $)^{4}$.

\section{Resultados}

Se efectuaron 2.096 atenciones oftalmológicas, con un promedio de edad de 71 años (Tabla 1), de las cuales $1.592(75,95 \%)$ correspondieron a errores refractivos, $415(19,89 \%)$ a cataratas, 226 $(10,81 \%)$ sospechas de glaucoma y $46(2,19 \%)$ a retinopatía diabética. Se resolvieron a nivel primario 1.766 pacientes, lo que da un índice de resolutividad global de $84,25 \%$; con $15,75 \%$ de derivación para resolución quirúrgica de patologías como cataratas (GES), uveítis, capsulotomías, iridotomías, entre otros (Tabla 2). En cuanto a los motivos de derivación, los pacientes con catarata, pterigion y obstrucción de vía lagrimal lo hicieron

\section{Tabla 1. Características demográficas generales}

\begin{tabular}{|lcc|}
\hline Detalle & n & \% \\
\hline Pacientes atendido & 2.096 & 100 \\
\hline Edad promedio & 71 años & \\
$\begin{array}{l}\text { Índice de resolutividad } \\
\text { promedio }\end{array}$ & 1.766 & 84,25 \\
Derivaciones* & 330 & 15,75 \\
\hline
\end{tabular}

*Cataratas GES, capsulotomías, iridotomías, uveítis, pterigiones y obstrucción de vías lagrimales. 
Tabla 2. Distribución de atenciones según patologías

\begin{tabular}{|lcl|}
\hline Detalle & n & Porcentaje \\
Vicios de refracción & 1.592 & $75,95 \%$ \\
\hline Cataratas & 415 & $19,89 \%$ \\
Cataratas con requisito GES & 181 & $\begin{array}{l}43,61 \% \text { del total de } \\
\text { cataratas }\end{array}$ \\
Glaucoma & 226 & $\begin{array}{l}10,81 \% \\
\text { Sospechas de glaucoma }\end{array}$ \\
& 163 & $\begin{array}{l}72,12 \% \text { del total de } \\
\text { glaucomas }\end{array}$ \\
Diagnóstico de glaucoma & 63 & $\begin{array}{l}27,88 \% \text { del total de } \\
\text { glaucomas }\end{array}$ \\
& 509 & $24,28 \%$ \\
Pacientes diabéticos & 46 & $9,04 \%$ de diabéticos \\
Retinopatía diabética & & \\
\hline
\end{tabular}

para resolución quirúrgica en centro terciario; las capsulotomías e iridotomías para realizar láser Yag, y las uveítis para evaluación por especialista y manejo inmunosupresor. En las Tablas 3 y 4 se detallan las atenciones según severidad de retinopatía diabética y subtipo de glaucoma respectivamente.

\section{Discusión}

Podemos observar que la gran mayoría de pacientes tratados poseían patologías susceptibles de tratar a nivel primario. El índice de resolutividad promedio fue de $84,25 \%$, acorde con el de otras UAPOs. Esto se debe principalmente a que la gran mayoría de las patologías tratadas fueron vicios de refracción $(75,9 \%)$ o patologías no quirúrgicas. Además, la posibilidad de contar, como lo es en la UAPO de Nuñoa, con un campo visual computarizado (Humphrey ${ }^{\circledR}$ Field Analyzer) gracias al cual los pacientes con sospecha de glaucoma pueden ser diagnosticados, tratados y controlados en la misma UAPO.

Se puede observar que las causas de las derivaciones desde la UAPO al nivel terciario consisten en procedimientos quirúrgicos como cirugías de cataratas, pterigiones y estrabismos, como también procedimientos menores ambulatorios como las capsulotomías e iridotomías, que pueden ser resueltas mediante láser. De llegar a tener un equipo para láser en la UAPO, la resolutividad
Tabla 3. Distribución según severidad de la retinopatía diabética $(n=46)$

\begin{tabular}{|lrr|}
\hline Retinopatía diabética & n & \% \\
\hline Leve & 16 & 34,78 \\
Moderada & 22 & 47,82 \\
Severa & 2 & 4,34 \\
\hline Proliferativa & 6 & 13,04 \\
\hline
\end{tabular}

Tabla 4. Distribución según subtipo de casos con diagnóstico de glaucoma $(n=63)$

\begin{tabular}{|lrr|}
\hline Glaucomas & n & \% \\
\hline Ángulo abierto & 51 & 80,95 \\
Ángulo cerrado & 2 & 3,17 \\
Secundario & 10 & 15,87 \\
\hline
\end{tabular}

sería aun mayor, derivándose solo aquellos que requieran ser operados en un pabellón. Nuestro trabajo muestra las tasas de prevalencia de patologías de tres comunas de Santiago, con un número no despreciable, lo que facilitaría la distribución de recursos que podría aportar el MINSAL a estas comunas en materia de salud visual. Una segunda etapa será realizar un estudio en el Hospital del Salvador objetivando el impacto que ha generado el tener oftalmólogos en las UAPOS en cuanto a sus listas de espera en las distintas patologías.

Es importante resaltar, junto con la edad promedio avanzada de los pacientes, la gran prevalencia de vicios de refracción. Esto resulta favorable, debido a que corresponden a patologías de baja complejidad, con alta probabilidad de tratamiento y baja tasa de derivación. Asimismo, al analizar patologías más complejas con riesgo de ceguera irreversible, como retinopatía diabética o glaucoma, un gran porcentaje con estadíos iniciales y con subtipo ángulo abierto, respectivamente, es decir, patologías de baja complejidad donde la unidad de atención primaria oftalmológica podría realizar su manejo y control.

\section{Conclusiones}

Las UAPOs han mejorado la cobertura y la eficiencia en la resolución de patologías prevalentes o con riesgo de ceguera, lo cual tendrá impacto 
en la salud visual pública chilena. Su trabajo se focaliza principalmente en la atención de una población con pocas oportunidades de acceso a la atención oftalmológica, poniendo énfasis en el nivel primario de atención en salud. A través de los años se ha ido trasformando en un modelo de alta resolutividad y eficiencia sanitaria. De nuestro trabajo se concluye que las UAPOs presentan altos índices de resolutividad, por lo cual creemos que el resultado del trabajo en red, entre el nivel primario y terciario, mediante protocolos de derivación y coordinación de servicios y recursos, tanto económicos como humanos, es y debe ser un ejemplo como modelo a seguir a nivel nacional y latinoamericano, para asegurar la cobertura y continuidad de la atención oftalmológica.

Como opciones de mejora, proponemos la incorporación del glaucoma al plan GES y de usuarios con patologías GES a las UAPOs mediante una gestión clínico-administrativa y atención en red que asegure una continuidad en la atención del paciente oftalmológico. En cuanto a la cobertura del fondo de ojo, se podría optimizar mediante la habilitación de las UAPOs con recurso de teleoftalmología, licitación centralizada del informe de teleoftalmología y la elaboración de protocolos entre UAPOs con el programa cardiovascular para atención integral de pacientes diabéticos. Finalmente, lograr una mejor coordinación entre el nivel primario y el Servicio de Oftalmología mediante un sistema informático coordinado para mejorar la continuidad de atención, optimizar los sistemas de referencia-contrarreferencia y capacitación del personal humano.

\section{Referencias}

1. Riesco B, Sáez V, Escobar S, Barría F, Donoso R, Gil C. Unidades de atención primaria en oftalmología en Chile: historia y funciones. Rev Med Chile 2015; [online] 143 (7): 919-24. Available at: http://www.scielo.cl/scielo. php?script=sci_arttext\&pid=S0034-98872015000700013 [Accedido el 5 de diciembre de 2017].

2. Guía clínica tratamiento quirúrgico de cataratas congénitas y adquiridas [Internet]. Minsal.cl. 2010 [citado el 4 de junio de 2018]. Available from: http://www.minsal. cl/portal/url/item/7220f6b9b0044176e04001011f0113b7. pdf

3. Guía clínica para el tratamiento farmacológico del glaucoma [Internet]. Minsal.cl. 2013 [citado el 4 de junio de 2018]. Available from: http://www.minsal.cl/portal/url/ item/dc4f32fad5975649e040010165012d1d.pdf.

4. Barría F, Martínez F. Guía práctica clínica de retinopatía diabética para Latinoamérica [Internet]. Iapb.org. 2011 [citado el 4 de junio de 2018]. Available from: https:// www.iapb.org/wp-content/uploads/Guia-Practica-Clinica-de-Retinopatia-Diabetica-para-Latinoamerica.pdf. 